Discrete Comput Geom 29:395-408 (2003)

DOI: $10.1007 / \mathrm{s} 00454-002-0755-6$

Geometry

\title{
New Bounds on the Katchalski-Lewis Transversal Problem
}

\author{
Andreas Holmsen \\ Department of Mathematics, University of Bergen, \\ Johannes Brunsgt. 12, 5008 Bergen, Norway \\ andreash@mi.uib.no
}

\begin{abstract}
In 1980 Katchalski and Lewis showed the following: if each three members of a family of disjoint translates in the plane are met by a line, then there exists a line meeting all but at most $k$ members of $\mathcal{F}$, where $k$ is some positive constant independent of the family. They also showed that $k$ can be taken to be less than 603 , and conjectured that $k=2$ is a universal bound for all such families. In 1990 Tverberg improved the upper bound by showing that $k \leq 108$ holds. We make further improvements on the upper bound of $k$, showing that $k \leq 22$. Finally, we give a construction of a family of disjoint translates of a parallelogram, each three being met by a line, but where any line misses at least four members. This provides a counterexample to the Katchalski-Lewis conjecture.
\end{abstract}

\section{Introduction}

Let $\mathcal{F}$ be a family of disjoint convex sets in the plane. A line meeting every member of $\mathcal{F}$ is called a common transversal of $\mathcal{F}$, and we say that $\mathcal{F}$ has property $T$. If every $n$-membered subfamily of $\mathcal{F}$ has a common transversal we say that $\mathcal{F}$ has $T(n)$. A family of disjoint convex sets that has $T(n)$ is called a $T(n)$-family. A transversal for a given family induces in the natural way an ordering of the family and this ordering, together with its reverse, is called a geometric permutation. If there exists a direction in the plane such that any line in this direction intersects at most one member of $\mathcal{F}$, we say that $\mathcal{F}$ is totally separable.

Over the years considerable effort has been devoted to finding general conditions on the family $\mathcal{F}$ such that for some integer $n, T(n)$ would imply $T$. For general families of convex sets, such an integer does not exist, but if one specializes the family, such results can be obtained. One such result is the following: For a family of disjoint translates of a compact convex set in the plane, $T(5) \Rightarrow T$. This long-standing conjecture by Grünbaum was proved by Tverberg [10]. 
Prior to Tverberg's proof, Katchalski et al. [9] had introduced geometric permutations (or GPs), a concept which has turned out to be very useful when working on transversal problems. Also before the proof of Grünbaum's conjecture, Katchalski and Lewis [8] asked the following question: What if one replaces 5 by 3 in Grünbaum's conjecture? The answer they found was that there always exists a transversal meeting all but some fixed amount of translates. They showed that this number could be taken to be less than $192 \pi$, but they remark that this is obviously too large. They also conjectured that the number 2 would suffice, i.e., for any $T$ (3)-family of disjoint translates there exists a transversal meeting all but at most two of the members. In 1990 Tverberg [11] remarked that the number can be taken to be less than 108, still a value which is considered much too large. In [2] Bezdek gave a construction of $n$ disjoint unit discs, $n \geq 6$, where each three have a transversal, and any line misses at least two of the discs. This example shows that the Katchalski-Lewis conjecture, if true, is best possible, meaning that one in general cannot expect to find a transversal meeting all but one member.

For a family $\mathcal{F}$ of convex sets, let $k$ be the smallest integer such that there is a transversal meeting all but at most $k$ of the members of $F$. We then say that $\mathcal{F}$ has $T-k$. Using this terminology, we can summarize what is known about the KatchalskiLewis transversal problem as follows. For any family of disjoint translates in the plane $T(3) \Rightarrow T-k$, where $2 \leq k \leq 108$. We shall improve the upper and lower bounds of $k$. The main result of this paper is the following.

Theorem 1. There exists a positive integer $k$, with $k \leq 22$, such that for any family of disjoint translates of a compact convex set in the plane, $T(3) \Rightarrow T-k$. Moreover, we must have $k \geq 4$.

The proof of the upper bound of Theorem 1 uses basically the same idea as the Katchalski-Lewis proof. That is, we find a certain line $\mathcal{L}$, and show that the translates not meeting $\mathcal{L}$ must lie in certain bounded regions. This is done in Section 6.

In Sections 4 and 5 we estimate how many translates can lie in the bounded regions.

In Section 2 we state some basic reductions that should be well-known to readers familiar with transversal problems. Section 3 introduces terminology and standard results that we need to state and prove our technical lemmas, and in Section 7 we construct an example showing the lower bound of Theorem 1.

\section{Basic Reductions}

Here we review some well-known reductions. The proofs are quite standard to anyone familiar with geometric transversal theory and are not given here.

First we may assume that $K$ is two-dimensional. Otherwise, if $K$ were a point or a segment it is easily seen that $T(3)$ would imply $T$. Next we may assume that $\mathcal{F}$ is finite, as for an infinite family of disjoint, congruent, compact, convex sets, $T(3)$ implies $T$ [4, Proposition 91].

The next reduction was noted by Tverberg [10] and has turned out to be quite useful. Let $K$ be a convex set and let $K^{\prime}$ be the centrally symmetric set obtained by letting $K^{\prime}=\frac{1}{2} K-\frac{1}{2} K$. If we express $\mathcal{F}$ as $\mathcal{F}=\left\{K+v_{i}, i \in I\right\}$, where the $v_{i}$ are the 
translation vectors, and we let $\mathcal{F}^{\prime}=\left\{K^{\prime}+v_{i}, i \in I\right\}$, then $\mathcal{F}^{\prime}$ is a family of translates, and it is easily seen that $\mathcal{F}$ and $\mathcal{F}^{\prime}$ share the same properties with respect to disjointness, transversals, and GPs. Thus we may assume that $K$ is a centrally symmetric convex set.

The properties of $\mathcal{F}$ that we are studying are clearly affine invariants, thus we may, for any given $K$, use an affinely equivalent $K_{a}$. For reasons that will become clear later (Section 5) we are interested in the translates being as "fat" as possible. Behrend [1] showed that any centrally symmetric compact convex set $K$ is affinely equivalent to a set $K_{a}$ with center at the origin, $K_{a} \subset D$, where $D$ is the disc of radius $\frac{1}{2}$ centered at the origin, and such that we have one of the following cases:

(1) $K_{a}$ has two boundary points $v_{i}$ and $v_{j}$ that lie on the boundary of $D$ such that the angle between $v_{i}$, the origin, and $v_{j}$ equals $\pi / 2$.

(2) $K_{a}$ has three boundary points $v_{i}, v_{j}, v_{k}$ that lie on the boundary of $D$ such that if we let $\alpha, \beta, \gamma$ be the angles between $v_{i}$ and $v_{j}, v_{j}$ and $v_{k}, v_{k}$ and $-v_{i}$, respectively, then $0<\alpha, \beta, \gamma<\pi / 2$.

Thus, we assume, throughout, that $K$ is of one of the forms described above, and we say that $K$ is standardized. Furthermore, if $K$ is of the first form we say that $K$ contains $a$ square, and if $K$ is of the second form we say that $K$ contains a hexagon. It also follows that the diameter of $K$ equals 1 and the area is at least $\frac{1}{2}$.

\section{Some Useful Results Concerning $T(3)$-Families}

Here we review some known results concerning $T$ (3)-families of translates. We only outline the proofs here, and we refer the reader to [3], [5], and [11] for the complete proofs.

The following observation was made by Tverberg [11], improving a basic result by Eckhoff [3].

Proposition 1. Let $\mathcal{F}$ be a $T$ (3)-family of disjoint translates. There exist two members $X$ and $Y$ of $\mathcal{F}$ such that each member of $\mathcal{F} \backslash\{X, Y\}$ meets the two parallel segments forming bdry $(\operatorname{conv}(X \cup Y)) \backslash(X \cup Y)$.

Sketch of Proof. Eckhoff [3] proved first that any T(3)-family of translates must contain two members $X$ and $Y$ such that each member meets the two parallel nonseparating common support lines for $X$ and $Y$.

Let $F=\left\{K+v_{i} ; i \in I\right\}$ be a family of translates of a compact convex set $K$. (Note that $K$ is not assumed to be centrally symmetric and the members of $F$ are not assumed to be disjoint.) For each pair $K+v_{i}, K+v_{j}$ of $F$ he lets $d_{i j}$ denote the distance between $v_{i}$ and $v_{j}$. Furthermore, he lets $p_{i j}$ denote the length of the orthogonal projection of $K$ onto a line orthogonal to the line through $v_{i}$ and $v_{j}$. For a member $K+v_{k} \in F$, he then lets $h_{i j}(k)$ denote the distance from $v_{k}$ to the line through $v_{i}$ and $v_{j}$.

Next he defines the function $f(i, j)=d_{i j} p_{i j}$, and lets $\{x, y\}$ be the pair of $I$ that maximizes $f$. 
Let $z \in I \backslash\{x, y\}$ and assume that $K+v_{z}$ does not meet either of the two common parallel support lines for $K+v_{x}$ and $K+v_{y}$, which implies that

$$
f(x, y)<l_{x y} h_{x} y(z)=l_{x z} h_{x z}(y)=l_{y z} h_{y z}(x) .
$$

He then proceeds to argue that for one of the pairs, $\{x, z\}$, say, we must have $h_{x z}(y)<p_{x z}$ or else $K+v_{x}, K+v_{y}, K+v_{z}$ would not have a transversal. However, this gives us

$$
f(x, y)<l_{x z} h_{x z}(y)<l_{x z} p_{x z}=f(x, z),
$$

contradicting the choice of $\{x, y\}$.

Later Tverberg [11] showed, by a simple shrinking argument, that the members of $F$ not only meet the two parallel support lines of $K+v_{x}$ and $K+v_{y}$, but that they in fact meet the two parallel segments forming bdry $\left(\operatorname{conv}\left(\left(K+v_{x}\right) \cup\left(K+v_{y}\right)\right)\right) \backslash\left(\left(K+v_{x}\right) \cup\right.$ $\left.\left(K+v_{y}\right)\right)$.

For a $T$ (3)-family of disjoint translates, we call the sets $X$ and $Y$ of Proposition 1 the Eckhoff sets, and the two open boundary segments are referred to as the Eckhoff segments.

The following is just a simple observation, and we have left the proof to the reader.

Proposition 2. A totally separable family of convex sets in the plane has at most one GP.

If $\mathcal{F}$ is some family of sets in the plane where every $n$-membered subfamily of $\mathcal{F}$ is totally separable, we say that $\mathcal{F}$ has property $S(n)$. The following was proved in Theorem 4.6 of [5].

Proposition 3. Let $\mathcal{F}$ be a $T$ (3)-family of disjoint translates that also has $S(3)$. Then $\mathcal{F}$ admits a common transversal.

Sketch of Proof. Start by considering a T(3)-family of four disjoint translates that do not have a common transversal. From Proposition 1 we know there must be two Eckhoff sets and since there is no transversal to all the sets, the two remaining ones must meet opposite Eckhoff segments. Next we find that up to symmetries and/or relabelings of the sets, if necessary, there are essentially three different ways the family can have the property $T$ (3). (Two families are considered equivalent if they have the same GPs.) By an analysis of each of the three possible cases we find that there must always be a triple which is not totally separable. In other words we find that for $T$ (3)-families of translates, $S(3)$ implies $T(4)$. Using Proposition 2 and the property $T(4)$ we can then show that there must be an ordering of the family such that each three members have a transversal intersecting them consistently with the ordering. By Hadwiger's transversal theorem there is a transversal to the entire family. (Note that in view of Tverberg's transversal theorem we need only consider families of size 5.) 


\section{Small Families of Translates}

The smallest families of translates that have $T$ (3) but not $T$ are the ones consisting of four members. In this section we obtain some technical results concerning such families.

Since the translates we are studying have been assumed to be centrally symmetric, we define the distance between two translates $d(A, B)$ as the distance between the symmetry centers.

We now note that if three translates of diameter 1 have a transversal and the pairwise distance between the centers is greater than $\sqrt{2}$, then the three translates are totally separable. This is easily seen for families of discs of diameter 1 , and for general translates just circumscribe each translate by a disc of diameter 1 .

Thus, by Proposition 3, if a $T$ (3)-family of centrally symmetric standardized translates is such that the pairwise distances between the members are greater than $\sqrt{2}$, then the family admits a transversal.

Now we have the following technical lemma.

Lemma 2. Let $\{A, B, C, D\}$ be a $T$ (3)-family of disjoint centrally symmetric translates of diameter 1 , and assume $d(A, B)$ and $d(B, C)$ are greater than $\sqrt{2}$. Further, assume we have the geometric permutation ( $A B C)$, and that there is no transversal for $A, B, C$, D. Then:

(i) $d(A, C)>2$.

(ii) If we have $(A B D),(A C D)$, and $(C B D)$ or $(B D C)$, then $d(C, D)<\sqrt{2}$.

(iii) If we have $(A D C),(A D B)$, and $(B C D)$ or $(B D C)$, then $d(B, D)<\sqrt{2}$.

Proof. Now (i) is easily verified for discs of diameter 1. For general translates one just circumscribes the sets by discs, and the result follows.

We prove (ii) and (iii) first for discs of diameter 1. Consider (ii). Note that $A$ and $D$ are the Eckhoff sets. Assume $A$ lies to the left of $C$ and that $B$ meets the upper segment of the boundary of $\operatorname{conv}(A \cup C)$. Without loss of generality we may assume that $A, B, C$ is a critical triple, i.e., $A, B, C$ admit a unique transversal. To see this we observe that $B$ and $C$ must meet the upper and lower Eckhoff segments, respectively. Now we move $A$ toward the left in the direction of the Eckhoff segments. This will not change any of the GPs, and since we move $A$ away from the other sets, the pairwise distances between $A$, $B$, and $C$ remain greater than $\sqrt{2}$. We keep moving $A$ until the triple $A, B, C$ becomes critical. Note that this is bound to happen sooner or later.

Let the critical transversal of $A, B$, and $C$ define the horizontal direction. We first consider the case where we have $(C B D)$. $D$ must meet the nonhorizontal separating tangent for $B$ and $C$. Now move $D$ upward in the direction determined by this separating tangent until the transversal inducing $(A C D)$ becomes critical. Note that $d(C, D)$ will increase (see Fig. 1, left).

We now show that $d(C, D)<\sqrt{2}$. We define the points $\mathcal{P}, \mathcal{Q}, \mathcal{R}, \mathcal{R}^{\prime}$ as follows. Let $\mathcal{P}$ be the midpoint between the centers of $A$ and $C, \mathcal{Q}$ the center of $C, \mathcal{R}$ the midpoint between the centers of $C$ and $D$, and $\mathcal{R}^{\prime}$ the intersection between the vertical line through the center of $C$ and the ascending separating tangent for $A$ and $C$. Note that $\mathcal{P}, \mathcal{R}$, and $\mathcal{R}^{\prime}$ all lie on the ascending separating tangent for $A$ and $C$. Since the nonhorizontal separating 

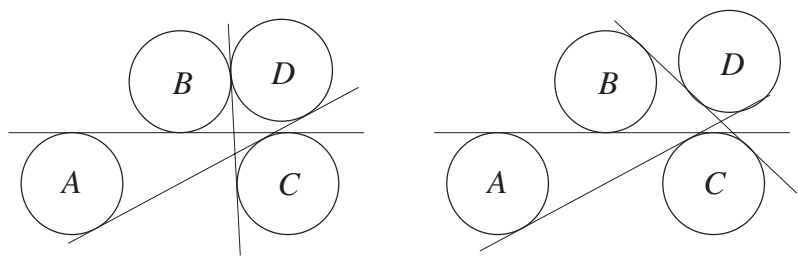

Fig. 1. Case (ii): left, $(C B D)$; right, $(B D C)$.

tangent for $B$ and $C$ must be descending it follows that $\mathcal{R}$ always lies between $\mathcal{P}$ and $\mathcal{R}^{\prime}$. Thus we have

$$
d(C, D)=2|\mathcal{Q R}|<2\left|\mathcal{Q} \mathcal{R}^{\prime}\right|<\frac{1}{\cos \left(\sin ^{-1}(1 / d(A, C))\right)}
$$

and by (i) we have that $d(A, C)>2$, which gives us $d(C, D)<2 / \sqrt{3}<\sqrt{2}$.

In the case where we have $(B D C)$ we note that $D$ must meet the upper segment of $\operatorname{conv}(B \cup C)$ as well as the ascending separating tangent for $A$ and $C$. Now it is easily seen that there must be a transversal inducing $(B D C)$ of slope between -1 and 0 , and that the slope of a transversal inducing $(A C D)$ must lie between 0 and $1 / \sqrt{3}$. This implies that the outer angle between the separating tangents for $C$ and $D$ is less than $\pi / 2$. From this it follows easily that $d(C, D)<\sqrt{2}$. This proves (ii) for discs of diameter 1 (see Fig. 1, right).

Now for (iii). Assume first that we have $(B C D)$. Without loss of generality we may assume that $A, B, C$ is a critical triple, and that $d(A, B)=\sqrt{2}$. To see this we move $A$ toward the left in the direction determined by the centers of $A$ and $B$. This will not change any of the GPs and since we move $A$ away from the rest of the sets, the pairwise distances between $A, B$, and $C$ remain greater than $\sqrt{2}$. We keep moving $A$ until the triple $A, B$, $C$ becomes critical. Now we move $A$ toward the right in the direction determined by the critical transversal for $A, B, C$. We keep moving $A$ until $d(A, B)=\sqrt{2}$. This will not change any of the GPs and since we have $(B C D), D$ must lie so far to the right that $A$ and $D$ will remain disjoint. Let the line through the centers of $A$ and $C$ be the $x$-axis and let the vertical line through the center of $B$ be the $y$-axis. Let $(x, y)$ be the center of $D$. Since we have $(B C D)$ it follows that we must have $x>0$, and since $D$ does not meet the unique transversal for $A, B, C$ we have $y<0$. Finally, $(A D B)$ implies $y \geq x-\sqrt{2}+1$. This is easily seen to imply that $d(B, D)<\sqrt{2}$ (see Fig. 2, left).

Finally, assume we have $(B D C)$. As above we may assume that the transversal for $A$, $B, C$ is critical, and let this determine the horizontal direction. It follows from the fact that $d(A, B)>\sqrt{2}$ that the lower segment of $\operatorname{conv}(A \cup B)$ has slope between 0 and 1 . Similarly, the lower segment of $\operatorname{conv}(B \cup C)$ has slope between -1 and 0 . Since $D$ must meet each of these segments, it follows that the outer angle of the separating tangents of $B$ and $D$ must be less than $\pi / 2$. This gives us $d(B, D)<\sqrt{2}$ (see Fig. 2, right).

Now (ii) and (iii) also hold for the general case, that is replacing the discs by centrally symmetric translates of diameter 1 . The arguments above hold the same, noting that if a general translate has a transversal inducing a certain GP, the family consisting of the circumscribed discs will also have a transversal inducing the same GP, save the cases 

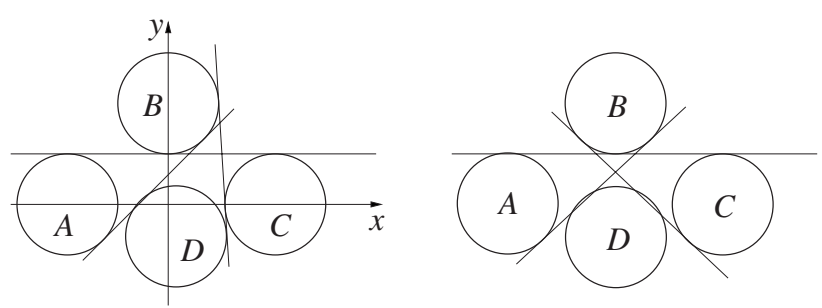

Fig. 2. Case (iii): left, $(B C D)$; right, $(B D C)$.

where the circumscribed discs are not disjoint (this can only occur if $d(X, Y)<1$ ). It is, however, easily seen that this last scenario does not cause any problems.

\section{Some Preliminary Bounds}

Let $K$ be a centrally symmetric standardized convex set and let $l$ be a support line of $K$. Let $l^{+}$and $l^{-}$denote the two opposite open half-planes separated by $l$ and suppose $K \subset l \cup l^{-}$. Let $K_{1}, K_{2}, \ldots, K_{m}$ be disjoint translates of $K$ such that $K_{i} \subset l^{+}$and $d\left(K, K_{i}\right)<\sqrt{2}$ for all $i=1, \ldots, m$. Now clearly $m$ must be bounded. In fact, we have the following:

Lemma 3. Let $K$ be a centrally symmetric standardized convex set, and let $l$ be a support line of $K$ such that $K \subset l \cup l^{-}$. If $K_{1}, \ldots, K_{m}$ are disjoint translates of $K$ such that $K_{i} \subset l^{+}$and $d\left(K, K_{i}\right)<\sqrt{2}$ for all $i=1, \ldots, m$, then $m \leq 7$.

Proof. We start with the easy case where the standardized $K$ contains a square. We may assume that the square contained in $K$ has edges parallel to the coordinate axes. Note that if $K$ and $K_{i}$ are separated by a strip parallel to an edge of the square of $K$, and of width equal to the width of the square, then $d\left(K, K_{i}\right)>\sqrt{2}$. By a rotation and/or reflection, if necessary, we may assume that the slope of $l$ is between 0 and 1 . We may also translate $l$ such that it becomes a support line of the square of $K$ rather than a support line of $K$, as the $K_{i}$ are still contained in $l^{+}$. Now consider the square lattice obtained from the square of $K$. Each $K_{i}$ must contain a lattice point, and it is easily verified that there are at most five lattice points that are such that a $K_{i}$ containing one of these lattice points would lie in $l^{+}$and $d\left(K, K_{i}\right)<\sqrt{2}$ (see Fig. 3).

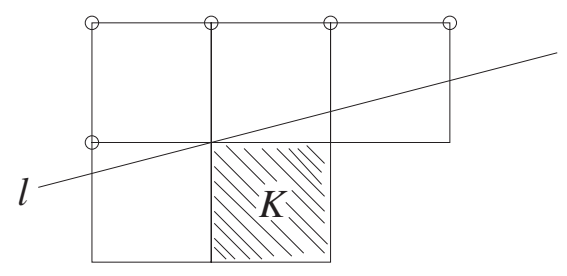

Fig. 3. $K$ contains a square. 


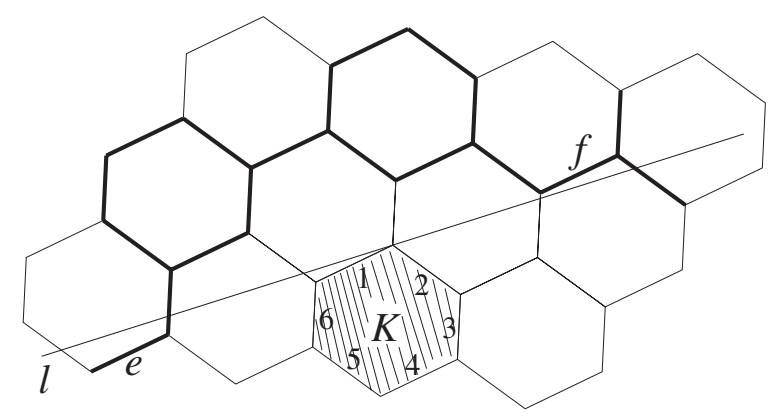

Fig. 4. $K$ contains a hexagon.

Now consider the case where the standardized $K$ contains a hexagon. Again if $K$ and $K_{i}$ are separated by a strip parallel to an edge of the hexagon, and of width equal to the width of the hexagon in this direction, then $d\left(K, K_{i}\right)>\sqrt{2}$. We may also assume that $l$ is a support line of the hexagon of $K$ rather than a support line of $K$, as the $K_{i}$ are still contained in $l^{+}$. Now consider the hexagonal lattice obtained from the hexagon of $K$. By symmetry we may assume that $l$ is a support line through the vertex determined by edges 1 and 2 of $K$ (see Fig. 4), and that the slope of $l$ is such that it lies between that of the line containing edge 1 of $K$ or the line containing the top vertex of the hexagon adjacent to edge 3 of $K$. Each $K_{i}$ must contain an edge of the lattice, and it is easy to verify that the possible edges that a $K_{i}$ can contain such that $d\left(K, K_{i}\right)<\sqrt{2}$ are the ones that are outlined in Fig. 4. It is also easy to see that the edges $e$ and $f$ cannot both be contained in $l^{+}$at the same time, as $e$ and $f$ are symmetric about the midpoint of edge 1 . Now two neighboring edges cannot be contained in distinct translates of $K$ as this would violate the disjointness. All that remains is therefore an easy check (left to the reader) that the graph of the outlined edges which lies entirely above $l$ contains at most seven pairwise disjoint edges.

Remark. Another way of approaching the problem of Lemma 3 would be to note that each $K_{i}$ must lie in the bounded region of $\mathcal{D} \cap l^{+}$where $\mathcal{D}$ is the disc of radius $\sqrt{2}+\frac{1}{2}$ centered at the center of $K$. As the $K_{i}$ are of area at least $\frac{1}{2}$, we find that

$$
n \leq\left\lfloor\frac{\text { Area of } \mathcal{D} \cap l^{+}}{\frac{1}{2}}\right\rfloor \leq 4 \int_{1 / 2 \sqrt{2}}^{\sqrt{2}+1 / 2} \sqrt{\left(\sqrt{2}+\frac{1}{2}\right)^{2}-x^{2}} d x .
$$

This gives us $n \leq 8$. The proof of the next lemma, however, uses this approach.

Lemma 4. Let $E$ be one of the Eckhoff sets of a T(3)-family of disjoint standardized translates and let $\mathcal{S}$ be one of the Eckhoff segments. If $K_{1}, \ldots, K_{n}(\neq E)$ are members such that every $K_{i}$ meets $\mathcal{S}$ and $d\left(E, K_{i}\right)<\sqrt{2}$ for every $i=1, \ldots, n$, then $n \leq 8$.

Proof. We may assume that the Eckhoff segments are horizontal and that $E$ is the left Eckhoff set. Let $\mathcal{C}$ be a central chord of $E$ that meets the Eckhoff segments. Since we have not specified whether $\mathcal{S}$ is the upper or lower Eckhoff segment, we may assume, by 
symmetry, that the slope of $\mathcal{C}$ is negative. Let $l$ be the length of $\mathcal{C}$ and let $s$ be the slope of $\mathcal{C}$. It is easily seen, since the translates are standardized, that we must have $l \in[1 / \sqrt{2}, 1]$ and $s \in[-\infty,-1]$. If we let $v$ be the angle between $\mathcal{C}$ and the Eckhoff segments, the width of the strip bounded by the lines containing the Eckhoff segments is $l \sin v$. Now if $\mathcal{S}$ is the upper (lower) Eckhoff segment, then every translate meeting $\mathcal{S}$ must lie in the closed strip bounded by the line containing the lower (upper) Eckhoff segment and the line that lies at distance $l \sin v$ above (below) the upper (lower) Eckhoff segment. Let $\mathcal{U}$ be this strip.

Let $\mathcal{L}$ be the left support line of $E$ which is parallel to $\mathcal{C}$. Since the translates are standardized the distance between $\mathcal{C}$ and the center of $E$ is at most $\frac{1}{2}$. Now every translate of the $T$ (3)-family must lie in the right half-plane bounded by $\mathcal{L}$. Let $\mathcal{V}$ be this half-plane. Since we are interested in the translates $K_{i}$ that meet $\mathcal{S}$ such that $d\left(E, K_{i}\right)<\sqrt{2}$, the $K_{i}$ 's must lie in the disc of diameter $\sqrt{2}+\frac{1}{2}$ centered at the center of $E$. Let $\mathcal{D}$ be this disc.

We have found that the $K_{i}$ 's must lie in the bounded region $\mathcal{U} \cap \mathcal{V} \cap \mathcal{D}$. We now divide the maximal area of this region by the minimal area of a standardized translate to finish the proof.

We now specify whether $\mathcal{S}$ is the upper or lower Eckhoff segment. Since we have chosen $\mathcal{C}$ to have negative slope, it is clear that the area of $\mathcal{U} \cap \mathcal{V} \cap \mathcal{D}$ is larger if $\mathcal{S}$ is chosen to be the upper Eckhoff segment. The area will also be increased by letting $d=1$ and assuming that the distance between $\mathcal{L}$ and the center of $E$ equals $\frac{1}{2}$. The only remaining variable is therefore $v$. Consider the vertical line through the center of $E$. This line divides $\mathcal{U} \cap \mathcal{V} \cap \mathcal{D}$ into two parts. It is easy to verify that the area of the left part is

$$
1+\sin v \cos v
$$

while the area of the right part is

$$
\int_{-3 / 2 \sin v}^{1 / 2 \sin v} \sqrt{\left(\sqrt{2}+\frac{1}{2}\right)^{2}-x^{2}} d x
$$

The maximum of the first function occurs for $v=\pi / 4$ while the maximum of the second function occurs for $v=\pi / 2$. Adding the sums of the respective maxima and dividing by the minimum area of a standardized translate, which is $\frac{1}{2}$, we find that at most nine translates can exist in $\mathcal{U} \cap \mathcal{V} \cap \mathcal{D}$, one of which is $E$. (Of course the sum of the maxima of the functions is greater than or equal to the maximum of the sum of the functions, but in this case the latter does not give a tighter bound!)

If we replace the strip $\mathcal{U}$ in the proof of Lemma 4 by the strip bounded by the line that lies at distance $l \sin v$ above the upper Eckhoff segment and the line that lies at distance $l \sin v$ below the lower Eckhoff segment, we obtain a bound for how many translates can lie within distance $\sqrt{2}$ of $E$ (regardless of which Eckhoff segment they meet).

We can obtain a crude upper bound of the area of this new region by simply multiplying the area found in the proof of Lemma 4 by $\frac{3}{2}$. This gives us the following:

Lemma 5. Let $E$ be an Eckhoff set of a $T$ (3)-family of disjoint standardized translates. If $K_{1}, \ldots, K_{q}(\neq E)$ are members such that $d\left(E, K_{i}\right)<\sqrt{2}$ for every $i=1, \ldots, q$, then $q \leq 14$. 


\section{Proof of the Upper Bound of Theorem 1}

We are now ready to prove the upper bound of Theorem 1 . Let $\mathcal{F}$ be a $T(3)$-family of disjoint standardized translates and assume that the Eckhoff segments are horizontal. Let $E$ be the left Eckhoff set. We may assume that each Eckhoff segment meets more than 22 sets, or else the upper bound is trivial.

First we shall see that there exists a triple $E, A, B$ that does not admit a horizontal transversal and where the pairwise distances are greater than $\sqrt{2}$. Disregarding the at most 14 translates that lie within distance $\sqrt{2}$ of $E$ (Lemma 5), let $A$ be the translate that is the highest among the ones meeting the upper Eckhoff segment. That is, such that the lower horizontal support line of $A$ meets the rest of the translates that meet the upper Eckhoff segment (except possibly the ones within $\sqrt{2}$ of $E$ ). By Lemma 3 there can be at most seven translates that lie below the lower horizontal support line of $A$ that lie within distance $\sqrt{2}$ of $A$. If there are no more translates below this lower support line of $A$, then this line meets all but at most $14+7=21$ translates $\mathcal{F}$, and we are finished. Thus there must exist a translate $B$ that lies below the lower horizontal support line of $A$ which is at distance more than $\sqrt{2}$ of $E$ and $A$.

We now do the following: Move $E$ toward the left in the horizontal direction to obtain $E^{\prime}$. We stop moving $E$ at the point where some triple $E^{\prime}, A, B$, whose pairwise distances are greater than $\sqrt{2}$, is about to lose its transversal ( $E^{\prime}, A, B$ becomes a critical triple). This is bound to happen sooner or later as we showed that $\mathcal{F}$ must contain at least one such triple. We may now assume the situation to be the following: $A$ meets the upper Eckhoff segment, $B$ meets the lower Eckhoff segment, and $E^{\prime}, A, B$ have a unique transversal, $\mathcal{L}$. Further, we may assume that $\mathcal{L}$ induces $\left(E^{\prime} A B\right)$. To see this note that if $\mathcal{L}$ induces $\left(E^{\prime} B A\right)$ we just reflect $\mathcal{F}$ about a horizontal line and relabel $A$ and $B$. The only remaining alternative is that $\mathcal{L}$ induces $\left(A E^{\prime} B\right)$. However, since $A$ and $B$ meet opposite Eckhoff segments it is easily seen that $E^{\prime}, A, B$ cannot be totally separable, contradicting the fact that the pairwise distance between the members is greater than $\sqrt{2}$.

After moving $E, \mathcal{F}$ may no longer be a $T(3)$-family because we may have lost transversals for triples where the pairwise distances are less than or equal to $\sqrt{2}$. However, $\mathcal{F} \backslash\left\{E^{\prime}\right\}$ is still a $T(3)$-family. Consider a translate $X$ such that the pairwise distances of $E, A, B, X$ are greater than $\sqrt{2}$. Then $E^{\prime}, A, B, X$ is a $T(3)$-family and the triples of $E^{\prime}, A, B, X$ must be totally separable. Thus it follows from Proposition 3 that $E^{\prime}, A, B, X$ have a transversal, and since $E^{\prime}, A, B$ have a unique transversal, it follows that $X$ must meet $\mathcal{L}$. Therefore, every translate that does not meet $\mathcal{L}$ must lie within distance $\sqrt{2}$ of $E$, $A$, or $B$. However, we can in fact say more about the situation. Let $X$ be a translate that does not meet $\mathcal{L}$. Then we must have one of the following cases: (1) the upper Eckhoff segment induces $(E X A),(2)$ the upper Eckhoff segment does not induce (EXA) and $X$ lies above $\mathcal{L}$, or (3) the upper Eckhoff segment does not induce $(E A X)$ and $X$ lies below $\mathcal{L}$.

Case (1). First, $X$ must lie above $\mathcal{L}$. To see this, assume the contrary. Then $X$ must meet the upper Eckhoff segment between the points where $E$ and $\mathcal{L}$ meet the upper Eckhoff segment. Since $\mathcal{L}$ separates $A$ from $X$ and $B,(X A B)$ is impossible, so we consider the other possibilities. We cannot have $(X B A)$ because then $B$ would meet the lower segment of $\operatorname{conv}(X \cup A)$, and it is easily seen that $B$ also meets the lower segment of $\operatorname{conv}(E \cup A)$, meaning that $E, A, B$ must have another GP apart from $(E A B)$, contradicting the fact 
that it is totally separable (Proposition 2). Similarly, if we have ( $A X B$ ), then $X$ must meet the left segment of $\operatorname{conv}(A \cup B)$, but then also $E$ would meet this segment, again implying more than one GP for $E, A, B$. Thus $X, A, B$ does not have a transversal. Therefore $X$ must lie above $\mathcal{L}$. Now we cannot have $(E B X)$ because this would mean that $B$ meets the lower segment of $\operatorname{conv}(E \cup X)$, and it is easily seen that this would force $B$ to meet $\operatorname{conv}(E \cup A)$ giving $(E B A)$. However, then $E, A, B$ would have more than one GP. Thus we have $(E X B)$ or $(X E B)$. If we have $(E X B)$, then we must have $d(E, X)<\sqrt{2}$ or $d(B, X)<\sqrt{2}$, or else $(E X B)$ should have become critical before $\left(E^{\prime} X Y\right)$. If we do not have $(E X B)$, we must have $(X E B)$ and it follows from Lemma 2 that $d(E, X)<\sqrt{2}$.

Case (2). We cannot have $(X E B)$ because this would mean that $E$ meets $\operatorname{conv}(X \cup B)$, and it is easily seen that this would force $E$ to meet $\operatorname{conv}(A \cup B)$ giving $(A E B)$. However, then $E, A, B$ would have more than one GP. Thus we have $(E X B)$ or $(E B X)$, and clearly we cannot have $(A B X)$ as $\mathcal{L}$ separates $B$ from $A$ and $X$. If we have $(E X B)$ it follows that we must have $d(E, X)<\sqrt{2}$ or $d(B, X)<\sqrt{2}$, or else $(E X B)$ should have become critical before $\left(E^{\prime} A B\right)$. If we do not have $(E X B)$, we must have $(E B X)$, and it follows from Lemma 2 that $d(B, X)<\sqrt{2}$.

Case (3). We shall show that $X$ must lie within distance $\sqrt{2}$ of $A$. First, if we have $(E A X)$, then $d(A, X)$ must be less than or equal to $\sqrt{2}$ or else it follows from Lemma 2 that $d(E, X)$ is also greater than $\sqrt{2}$ and $E, A, X$ should have become critical before $E^{\prime}, A, B$. We must therefore check what happens if we have $(A E X)$ or $(E X A)$. Since $\mathcal{L}$ separates $A$ from $B$ and $X$ we cannot have $(B A X)$. If we have $(A E X)$ and $(A B X)$, then clearly $E$ and $B$ must meet opposite segments of $\operatorname{conv}(A \cup X)$ or else $E, A, B$ will have more than one GP. However, if $E$ and $B$ meet opposite segments, it is easily seen that they cannot be totally separable. Nevertheless, any of the other combinations of GPs is covered by Lemma 2, and thus $d(A, X)$ is less than or equal to $\sqrt{2}$.

Summarizing, we have the following: Let $X$ be a translate that misses $\mathcal{L}$. If $X$ lies above $\mathcal{L}$, then it must either lie within distance $\sqrt{2}$ of $E$ and meet the upper Eckhoff segment, or it must lie within distance $\sqrt{2}$ of $B$. If $X$ lies below $\mathcal{L}$, then it must lie within distance $\sqrt{2}$ of $A$. Thus Lemmas 3 and 4 give us that there can exist at most $8+7+7=22$ translates that do not meet $\mathcal{L}$. This proves the upper bound of Theorem 1 .

\section{Proof of the Lower Bound of Theorem 1}

We shall construct a family of translates with properties $T$ (3) and $T-4$, but not $T-3$. Let $K$ be a square with sides parallel to the coordinate axes. Let $A, B, C, D, E, X$ be translates of $K$, and let their relative positions be given as illustrated in Fig. 5.

Now let $A^{\prime}, B^{\prime}, C^{\prime}, D^{\prime}, E^{\prime}, X^{\prime}$ be the reflections of $A, B, C, D, E, X$, respectively, about a vertical line to the left of $A$. By appropriately moving the sets up or down, we can obtain $T$ (3). The resulting family is illustrated in Fig. 6. For convenience we have chosen to scale the $y$-axis appropriately. The translates therefore do not look like squares. We leave the verification of $T(3)$ to the reader.

We now show that the family does not have $T-3$. To do this we refer to the following theorem by Hadwiger-Debrunner (see Proposition 25 of [4]): If each three rectangles 


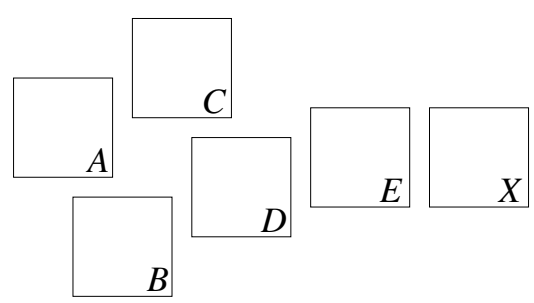

Fig. 5. First step of the construction.

of a family of parallel rectangles are intersected by an ascending line, then there is an ascending line that intersects all the rectangles of the family.

Assume there exists a transversal that meets all but at most three translates. It is easy to see that such a transversal cannot be parallel to the $x$-axis. We may therefore, by symmetry, assume that this transversal is ascending. If a pair of translates only admit descending transversals, we call this a descending pair. We define descending triples similarly. Our construction consists of the following descending pairs:

$$
\left\{C^{\prime}, B\right\} \quad\left\{C^{\prime}, D\right\} \quad\left\{A^{\prime}, B\right\}
$$

Every triple that contains a descending pair will naturally be a descending triple. Apart from the triples that contain a descending pair, it is easily verified that we have,

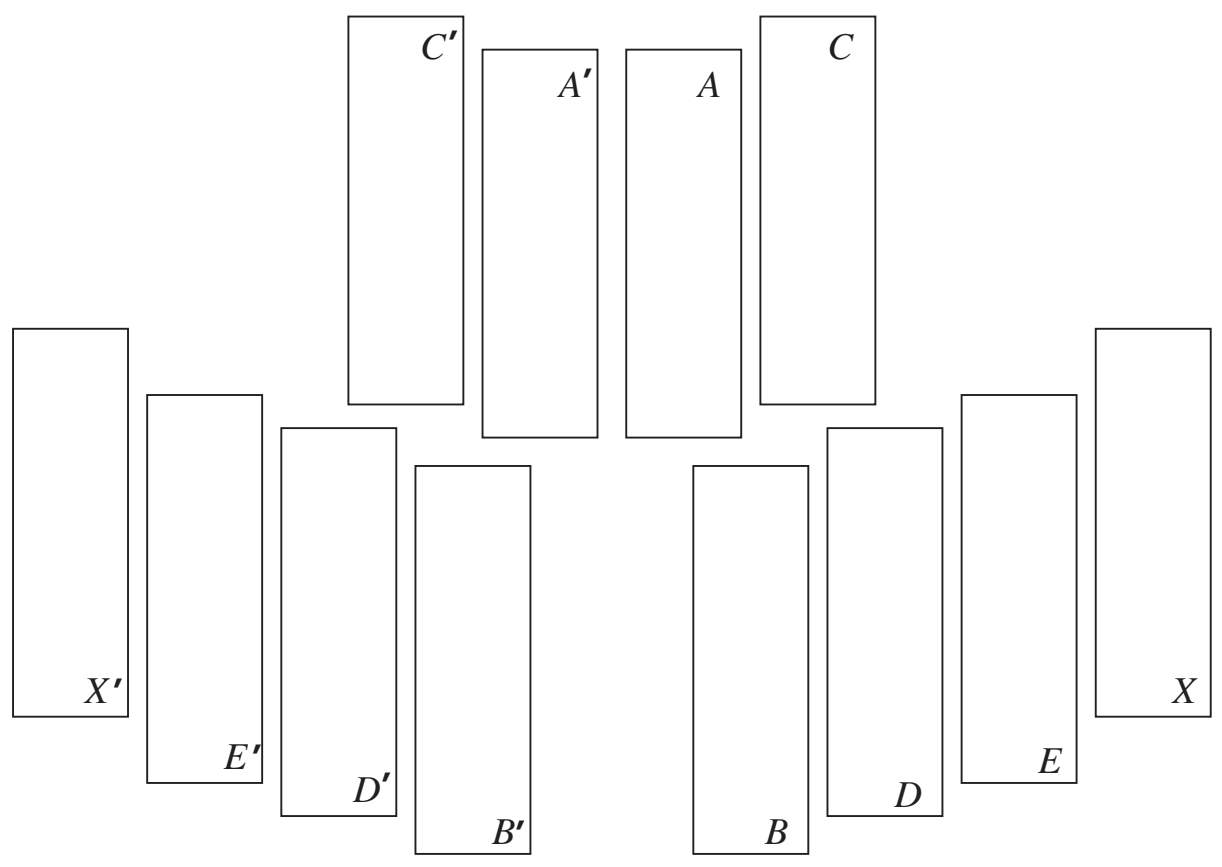

Fig. 6. $T(3) \nRightarrow T-3$. 
among others, the following descending triples:

$$
\begin{array}{ccc}
\{A, B, D\} & \{A, B, E\} & \left\{A, B, X^{\prime}\right\} \\
\{C, D, E\} & \left\{C, D, X^{\prime}\right\} & \left\{C^{\prime}, D^{\prime}, E\right\} \\
\{A, C, D\} & \left\{X^{\prime}, C^{\prime}, B^{\prime}\right\} & \left\{X, C^{\prime}, B^{\prime}\right\} \\
\left\{A^{\prime}, B^{\prime}, E\right\} & \left\{C^{\prime}, B^{\prime}, E\right\} & \left\{A^{\prime}, C, D\right\} \\
\left\{A^{\prime}, B^{\prime}, D\right\} & \{B, C, E\}
\end{array}
$$

Now, if there exists an ascending transversal that meets all but at most three translates, then by Hadwiger-Debrunner's theorem, we can remove three translates and thus "destroy" all the descending triples.

Given a family of sets $X=\left\{S_{1}, \ldots, S_{k}\right\}$ and a set $Y$, we say that $Y$ represents $X$ if for each $S_{j} \in X$ there is a $y \in Y$ such that $y \in S_{j}$. So what we want to show is that the descending triples cannot be represented by less than four elements.

We can start by concentrating on removing elements so that we destroy the descending pairs. For if we still have a descending pair, after removing three elements, we cannot have an ascending transversal that meets the remaining translates. It is easily verified that we must remove at least two translates to destroy the descending pairs, and that every triple representing the descending pairs contains at least one of the following pairs: $\left\{C^{\prime}, B\right\},\left\{C^{\prime}, A^{\prime}\right\}$, or $\{B, D\}$. However, these pairs represent the descending pairs, and it therefore suffices to check what happens when these pairs have been removed.

We start by removing the pair $\left\{C^{\prime}, B\right\}$. We are then left with the following descending triples:

$$
\begin{array}{rll}
\{C, D, E\} & \left\{C, D, X^{\prime}\right\} & \{A, C, D\} \\
\left\{A^{\prime}, B^{\prime}, E\right\} & \left\{A^{\prime}, B^{\prime}, D\right\} & \left\{A^{\prime}, C, D\right\}
\end{array}
$$

Removing the pair $\left\{C^{\prime}, A^{\prime}\right\}$, we are then left with the following descending triples:

$$
\begin{array}{lll}
\{A, B, D\} & \{A, B, E\} & \left\{A, B, X^{\prime}\right\} \\
\{C, D, E\} & \left\{C, D, X^{\prime}\right\} & \{A, C, D\} \\
& \{B, C, E\} &
\end{array}
$$

Finally, if we remove the pair $\{B, D\}$, we are left with the following descending triples:

$$
\begin{gathered}
\left\{X, C^{\prime}, B^{\prime}\right\} \quad\left\{X^{\prime}, C^{\prime}, B^{\prime}\right\} \quad\left\{C^{\prime}, D^{\prime}, E\right\} \\
\left\{A^{\prime}, B^{\prime}, E\right\} \quad\left\{C^{\prime}, B^{\prime}, E\right\}
\end{gathered}
$$

It is easily verified that in each of the cases above, the descending triples are not representable by a single element. Thus, the family does not satisfy $T-3$. Clearly, a horizontal line can be found that induces $\left(X^{\prime} E^{\prime} C^{\prime} A^{\prime} A C E X\right)$, thus the family satisfies $T-4$. Note that it is possible to add translates to the left of $X^{\prime}$ and the right of $X$, thus extending the family to arbitrary size. 


\section{Final Remarks}

The upper bound of Theorem 1 is, undoubtedly, still too large. In fact, the bounds of Lemmas 3 and 4 should be possible to improve. With the additional condition of $T$ (3) in Lemmas 3 and 4, it seems plausible that we should get something like $m, n \leq 3$. If true, this would give us $T(3) \Rightarrow T-9$, but we believe that even a better bound should be possible.

It should also be mentioned that better bounds have been obtained for special families of translates. Here we mention two examples.

If we let $K$ be a unit disc, Kaiser [7] has proved $T(3) \Rightarrow T-12$. His methods are different from ours, and work very well for the case of discs. However, for general $K$ the method does not seem to yield a better bound than ours.

If we let $K$ be a parallelogram, the present author [6] has proved $T(3) \Rightarrow T-4$. In view of the example showing the lower bound of Theorem 1, this result is the best possible. The first part of the proof is essentially the same as the method of this paper, and we obtain a line $l$ inducing $T-5$. The second part of the proof involves a series of rotations of $l$ about certain specified points, and we show that sooner or later the rotated line will induce $T-4$.

\section{References}

1. F. Behrend, Über einige Affininvarianten konvexer Bereiche. Math. Ann., 113 (1937), 713-747.

2. A. Bezdek, On the transversal-conjecture of Katchalski and Lewis, in Intuitive Geometry (Szeged, 1991), pp. 23-25. North-Holland, Amsterdam, 1994.

3. J. Eckhoff, Transversalenprobleme in der Ebene. Arch. Math., 24 (1973), 195-202.

4. H. Hadwiger, H. Debrunner, and V. Klee, Combinatorial Geometry in the Plane. Holt, Rinehart and Winston, New York, 1964.

5. A. Holmsen, Geometric Transversals for Families of Disjoint Translates in the Plane. Technical report, Department of Mathematics, University of Bergen, 2000.

6. A. Holmsen, A transversal theorem in the plane. In preparation, 2001.

7. T. Kaiser, Line transversals to unit disks. Discrete Comput. Geom., 3 (2002), 379-388.

8. M. Katchalski and T. Lewis, Cutting families of convex sets. Proc. Amer. Math. Soc., 79 (1980), $457-461$.

9. M. Katchalski, T. Lewis, and J. Zaks, Geometric permutations for convex sets. Discrete Math., 54 (1985), 271-284.

10. H. Tverberg, Proof of Grünbaum's conjecture on common transversals for translates. Discrete Comput. Geom., 4 (1989), 191-203.

11. H. Tverberg, On geometric permutations and the Katchalski-Lewis conjecture on partial transversals for translates, in Papers from the DIMACS Special Year on Discrete and Computational Geometry (New Brunswick, NJ, 1989/1990), pp. 351-361. American Mathematical Society, Providence, RI, 1991.

Received October 17, 2001, and in revised form October 17, 2002, and October 20, 2002.

Online publication January 30, 2003. 